\title{
Typing of Mycobacterium tuberculosis strains isolated in Community Health Centers of Rio de Janeiro City, Brazil
}

\author{
Maraníbia C Oelemann, Amanda NB Fontes, Marcia A da Silva Pereira, \\ Yolanda Bravin*, Guida Silva**, Wim Degrave, Anna Cristina C Carvalho***, \\ Rossana C Brito***, Afrânio L Kritski***, Philip N Suffys/ ${ }^{+}$
}

\author{
Departamento de Micobacterioses, Instituto Oswaldo Cruz-Fiocruz, Av. Brasil 4365, 21045-900 \\ Rio de Janeiro RJ, Brasil *Laboratório Central Noel Nutels, Rio de Janeiro RJ, Brasil **Secretaria Municipal de Saúde do Rio de \\ Janeiro, Rio de Janeiro, RJ, Brasil ***Programa Acadêmico em Tuberculose, Rio de Janeiro, RJ, Brasil
}

Fingerprinting of Mycobacterium tuberculosis strains from tuberculosis (TB) patients attended in Community Health Centers (CHCs) of Rio de Janeiro was performed to verify possible risk factors for TB transmission. A prospective community-based study was performed during the period of July 1996 to December 1996 by collecting sputum samples of 489 patients in 11 different CHCs in four different planning areas (APs) of the city. Bacteriological, clinical, and epidemiological information was collected and $\mathrm{M}$. tuberculosis genotypes defined after restriction fragment length polymorphism (IS6110-RFLP) and double repetitive element (DRE) fingerprinting of RFLP-clustered cases. Risk factors for TB transmission were looked for using three levels of cluster stringency. Among 349 (71\%) positive cultures obtained, IS6110-RFLP typing could be performed on strains from 153 different patients. When using identity of RFLP patterns as cluster definition, 49 (32\%) of the strains belonged to a cluster and none of the clinical or epidemiologic characteristics was associated with higher clustering levels. However, higher clustering level was observed in the AP including the central region of the city when compared to others. This strongly suggests that more recent transmission occurs in that area and this may be related with higher incidence of TB and HIV in this region.

Key words: tuberculosis - molecular epidemiology - IS6110-restriction fragment length polymorphism - double repetitive element

It is generally accepted that one third of the world population is infected with Mycobacterium tuberculosis (Mtb), and the estimated mortality because of tuberculosis (TB) in 2004 was nearly 2 million, demonstrating that Mtb is among the most deadly human infectious agents. The majority (95\%) of TB cases occur in developing countries, a scenario mainly responsible for negligence of the seriousness of the disease in richer countries. The latter however were obliged to reconsider the importance of the disease, due to the association between increased TB risk and HIV infection and the spreading of multi-drug resistant (MDR) strains all over the world (WHO 2006).

In developed nations, epidemiologic investigations that incorporate genotyping of Mtb by restriction fragment length polymorphism (RFLP) have lead to novel information about the spread of Mtb in the community and during institutional outbreaks, to a better understanding of the transmission dynamics of TB and to the differentiation of (re)infection from reactivation (Barnes \& Cave 2003, Seidler et al. 2004).

Since the early nineties, definition of risk factors for TB transmission in high TB incidence areas has been complemented by information obtained by Mtb geno-

Financial support: CNPq grants 5211 30/95-6 and 52 11 52/98-4 +Corresponding author: psuffys@ioc.fiocruz.br

ABF, MASP, PNS are CNPq fellowship

Received 9 October 2006

Accepted 12 March 2007 typing, the latter based on the recognition that clusters of fingerprints are a measure of recent transmission (Palittapongarnpim et al. 1997, Pineda-Garcia et al. 1997, Dale et al. 1999, Haas et al. 1999, Fandinho et al. 2000, Ferrazoli et al. 2000, Bruchfeld et al. 2002, Ferdinand et al. 2003, Easterbrook et al. 2004, Das et al. 2005). Mostly however, a convenient, and not a population-based and therefore adequate sampling, has been submitted to genotyping (Wilkinson et al. 1997, Van Rie et al. 1999, Godfrey-Faussett et al. 2000, Narayanan et al. 2002, ChanYeung 2003, Verver et al. 2004). Nonetheless, adding genotyping data, to those obtained during more traditional epidemiologic approaches, has revealed that particular Mtb strains can be responsible for MDR-outbreaks in the community, can disseminate more quickly or induce more severe forms of TB and can be responsible for failure or relapse during and after treatment (Lan et al. 2003).

In Brazil, TB remains an important public health problem, the country ranking 16th among the 22 countries with the highest TB burden worldwide. These high-burden countries account for approximately $80 \%$ of the estimated number of new TB cases (all forms) arising each year (WHO 2006). Together with Peru, Brazil accounted for 50\% of all TB cases in Latin America in 2002. The high prevalence of TB in Southeast Brazil is presumed to be a result of worsening living conditions in the urban areas, the HIV epidemic, inadequate diagnosis and treatment and inadequate use of resources. In this region, Rio de Janeiro, the countries' second largest city, presents approximately 9000 new cases per year, an average incidence rate of 175/100,000 (1998-2002) and a mortality rate of 12 per 100,000 inhabitants, among the high- 
est rates in the country (Cavalcante et al. 2003). Some strategies to revert this picture were induced, including the use of Directly Observed Therapy, Short-Course (DOTS) TB strategy in 1998 and provision of free antiretroviral therapy (HAART) for all diagnosed HIV cases in 1997 (Ministry of Health BNSAp. 2004, WHO 2005).

To our knowledge, only one study on the contribution of Mtb genotyping to evaluate TB detection of transmission, in the general population in Brazil, has been reported, namely in the City of São Paulo (Telles et al. 2005). We here present the use of IS6110-RFLP typing and DRE-PCR for cluster confirmation in an attempt to estimate the risk factors and regions for transmission of TB in the city of Rio de Janeiro, in a period before the DOTS and HAART implementation.

\section{MATERIALS AND METHODS}

Study location and population - Diagnosis and treatment of patients within the TB control program of Rio de Janeiro, a city with 5.6 million inhabitants in 1996, occurs in 28 Community Health Centers (CHCs), 48 general hospitals, two specialized hospitals for TB treatment, and two prisons. These institutions are localized in different areas of the city, divided into ten major planning areas (AP; Fig. 1), each covered by at least two CHCs, responsible for attending persons with suspicion of TB that are usually living or working in that region. Annually, $6000 \mathrm{~TB}$ cases are attended in CHCs and 3300 in hospitals or prisons. The number of individuals included in the bacteriogical evaluation for each $\mathrm{CHCs} / \mathrm{AP}$ was convenient and not strictly population-based.

From July to December 1996, individuals older than 14 years with suspicion of TB, and being attended at one of the 11 CHCs of AP1, AP2, AP3 or AP5 were considered for inclusion in the study, independent of sex, race, socioeconomic class, and HIV status. TB was diagnosed according to recommendations by WHO (2005) and at time of diagnosis, after written consent, a blood sample and two consecutive sputum samples were collected. The blood samples were stored at $-20^{\circ} \mathrm{C}$ until further analysis. An individual was considered a TB patient when positive culture for Mtb or two sputum smears positive for acid-fast bacilli (AFB+) were obtained. Smear negative pulmonary TB cases had at least two smear examinations negative for AFB, radiographic abnormalities consistent

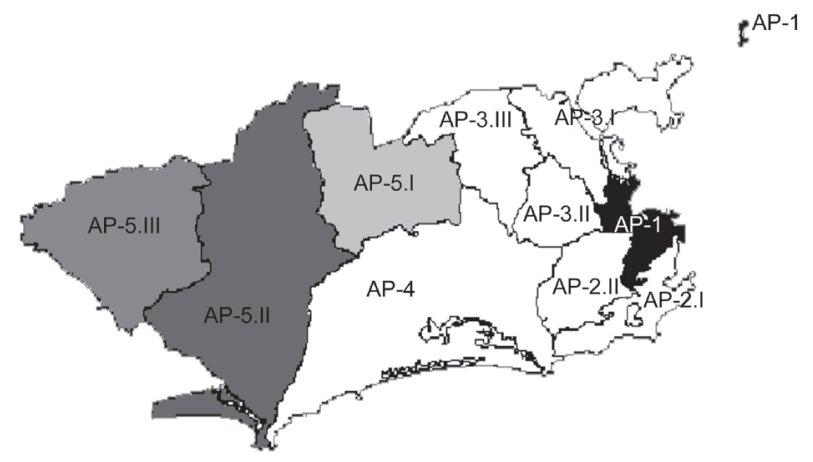

Fig. 1: map of the City of Rio de Janeiro with the different "Programatic Areas" for tuberculosis control. with active pulmonary TB, no response to a course of broad-spectrum antibiotics, decision by a clinician to treat with a full course of anti-TB therapy, or positive culture but negative AFB sputum examinations.

The site coordinator was responsible for collecting demographic, epidemiological, clinical, and laboratory data. Subjects were asked to answer a standardized questionnaire that comprised information regarding potential risk factors for TB infection such as sexual preference, living conditions (including slums), attendance at a hospital, education level, sex, permanence in a prison or police station, clinical form of TB, earlier treatment, intravenous drug use, drug resistance, HIV infection, and presence of a BCG scar. All clinical samples were sent to the Central Laboratory of Health Noel Nutels (Lacen, $\mathrm{RJ})$ for laboratory analysis.

This study was approved by the Institutional Review Boards of the Clementino Fraga Filho University Hospital of the Federal University of Rio de Janeiro and by those of each participating institution.

Specimen collection, culture collection, diagnostics, and drug sensitivity testing - Infection with HIV-1 and HIV-2 was verified by serology (Organon Teknica, Boxtei, The Netherlands) and positive cases were confirmed by Western blotting (Dupont, Wilmington, DE, US). Sputum was processed using the acetyl-cysteine method and samples were submitted to bacilloscopy according to Ziehl-Nielsen and culture in Löwenstein-Jensen medium (L-J), following the recommendations of the Brazilian National TB Control Program (Brasil 1994). Positive cultures were submitted to standard identification procedures for differentiation of species belonging to the Mtb Complex (MTBC) from atypical mycobacteria (Kent $\&$ Kubica 1985). Isolates were tested for susceptibility to isoniazid, rifampin, ethambutol, pyrazinamide, and streptomycin, using the proportion method (Brasil 1994, Kent \& Kubica 1985).

DNA fingerprinting - Cultures on L-J were submitted to DNA extraction and IS6110-RFLP typing according to a standardized methodology as described by van Embden et al. (1993). In each gel and each sample respectively, DNA from the reference strain Mt14323 and internal molecular weights markers for IS6110-containing fragment position normalization were included. The RFLP patterns were introduced into the GelCompar ${ }^{\circledR}$ software (Windows version 4.0; Applied Maths, Kortrijk, Belgium) and a similarity matrix and dendrogram were constructed using the Dice similarity coefficient and the UPGMA algorithm, using a position tolerance of $1.2 \%$. Clusters of IS6110-RFLP, indicated by GelCompar, were visually verified for consistency.

The majority of samples characterized by IS6110RFLP were also submitted to "Double Repetitive Element" PCR (DRE-PCR) fingerprinting as described by Montoro et al. (1998). Briefly, $2 \mu \mathrm{l}$ of each sample was added to a PCR mixture containing $20 \mathrm{mM}$ of each dNTP, $6 \%$ dimethyl sulfoxide, $1.25 \mathrm{U}$ Taq polymerase, and 100 ng of primers Ris1, Ris2, Pntb1, and Pntb2 in a total volume of $50 \mu$ l. The samples were amplified by thermal cycling during $1 \mathrm{~min}$ at $95^{\circ} \mathrm{C}, 2 \mathrm{~min}$ at $56^{\circ} \mathrm{C}$, and $1 \mathrm{~min}$ 
at $72^{\circ} \mathrm{C}$ and a final cycle of $7 \mathrm{~min}$ at $72^{\circ} \mathrm{C}$. Amplified products were verified by gel electrophoresis in $2 \%$ agarose and ethidium bromide staining and a 50 bp ladder was added to each gel as molecular weight marker.

Cluster definition - For cluster definition, three different stringencies were applied, including, ranging from more to less stringent: (i) identical IS6110-RFLP and DRE patterns, (ii) identical IS6110-RFLP patterns, and (iii) IS6110-RFLP patterns with $90 \%$ similarity on the dendrogram. Due to the sampling characteristics and typing efficiency in the present study, we applied the n-method for cluster degree calculation (Murray \& Alland 2002).

Statistical analysis - Data were analyzed using Epi Info (version 6.03, CDC, Atlanta, GA, US; public domain). Statistical significance of levels of association of cluster and non-cluster with epidemiologic and demographic characteristics for categorical variables were compared by the Fisher exact or chi-squared test. Continuous variables were compared by the Student t-test or the KruskalWallis rank-sum test. A confidence internal (CI) of $95 \%$ was used in all odds ratio (OR) calculations.

\section{RESULTS}

Patients and specimen collection - From July to December 1996, 859 TB cases were attended in 11 CHCs. Excluded from further analysis were 295 (34\%) cases that did not live in Rio de Janeiro, 67 (7.8\%) patients that had extrapulmonary TB, $48(5.5 \%)$ individuals that refused to participate in the study, and $26(3 \%)$ cases that had been using anti-TB drugs for more than 15 days. No statistically significant difference was observed regarding sex, age, and history of anti-TB treatment between included or excluded cases (data not shown). Sputum samples were collected from 438 patients $(51 \%)$ with available clinical, demographic, and epidemiological data. Serology for HIV was performed in 309 of these patients; positive results were confirmed in 27 cases $(8.7 \%)$ and a higher proportion of HIV positivity $(12.3 \%)$ observed in AP1 in comparison to other APs (median of 8.2\%).

Cultures and drug susceptibility patterns - Positive cultures for Mycobacterium were obtained from 349 different patients $(82 \%)$ and all were identified as belonging to the MTBC. Among these, 236 strains were submitted to susceptibility testing; the proportions of primary and acquired drug resistance were respectively 7.1 and $24.1 \%$. MDR, as defined by resistance to at least INH and RIF was detected in isolates from two patients that had a history of previous TB treatment.

Fingerprinting - Good quality IS6110-RFLP patterns were obtained from cultures of 153 patients, $47 \%$ of the culture positives TB cases. As demonstrated in Fig. 2, Mtb genotypes belonged to a limited number of groups as defined by the UPGMA-based dendrogram. Furthermore, genotypes presented between two and 22 copies of IS6110 with the majority (56\%) having between eight and ten copies; only two strains had less than six IS6110 copies. Forty nine of the isolates $(32.7 \%)$ presented a pattern that belonged to one of 21 clusters, including one cluster containing five isolates, five with three, and 15 with two samples.

Upon decreasing stringency of cluster definition to $90 \%$ similarity of RFLP patterns in the dendrogram, 28 clusters containing 72 strains $(45.1 \%)$ were observed. Among strains submitted to RFLP, DRE-PCR typing could be performed on 136 (data not shown), including 43 of the $49(88 \%)$ RFLP clustered strains. Among the latter, $34(79 \%)$ also had clustered DRE-PCR profiles but three RFLP clusters of two strains and three strains of the biggest RFLP cluster were discriminated by DREPCR. In the RFLP clusters with two strains, in each case, a single band difference was observed in the DRE pattern. Among the DRE patterns observed in strains forming the biggest RFLP cluster, four had a prominent band in common and two of these had an extra band on of different size; the fifth strain had an unrelated pattern (data not shown). The same DNA preparations were used for both techniques so sample mixup is unlikely to be the reason for this observation.

Association of patient and strain characteristics with genotype clustering - The clinical, epidemiological, and demographic characteristics of the patients, strain characteristics and cluster frequency as defined by identical RFLP patterns is presented in Table I. No statistically significant association between cluster level and any of these characteristics was observed. When other stringencies were used for cluster definition, again, no significant association of clustering with any characteristic was observed (data not shown).

To verify whether there was any relationship between the origin of the patients and recent $\mathrm{TB}$ transmission level, cluster frequency was determined for each AP separately. Significantly more clustering was observed among strains from patients attended in AP1 when compared to the others when using identical RFLP patterns for cluster definition (Table II). Interestingly, this difference continued statistically significant when increasing stringency of cluster definition (data not shown). To verify whether cluster frequency in the APs was directly related to the fraction of genotyped strains among those from all patients diagnoses in the different APs, both frequencies were compared, and as demonstrated in Table II, no direct relation was observed between both.

\section{DISCUSSION}

Investigation of TB transmission employing genotyping of Mtb as an additional tool was initially performed in some large cities of the United States (Small et al. 1994, Munsiff et al. 2003) and Europe (Lambregts-van Weezenbeek et al. 2003). These studies demonstrated the important contribution of recent TB transmission in many new TB cases, even in areas of low incidence, where TB was considered to be a controlled disease. Fingerprinting of Mtb also allowed better recognition of nosocomial and community outbreaks and of unsuspected or intricate chains of transmission (Caminero et al. 2001, Barnes \& Cave 2003, Seidler et al. 2004). Genotyping of Mtb strains also led to the visualization of the regionassociated population structure of the bug and of differences in strain variability and prevalence, as occurring both on a small and large scale. A considerable number 


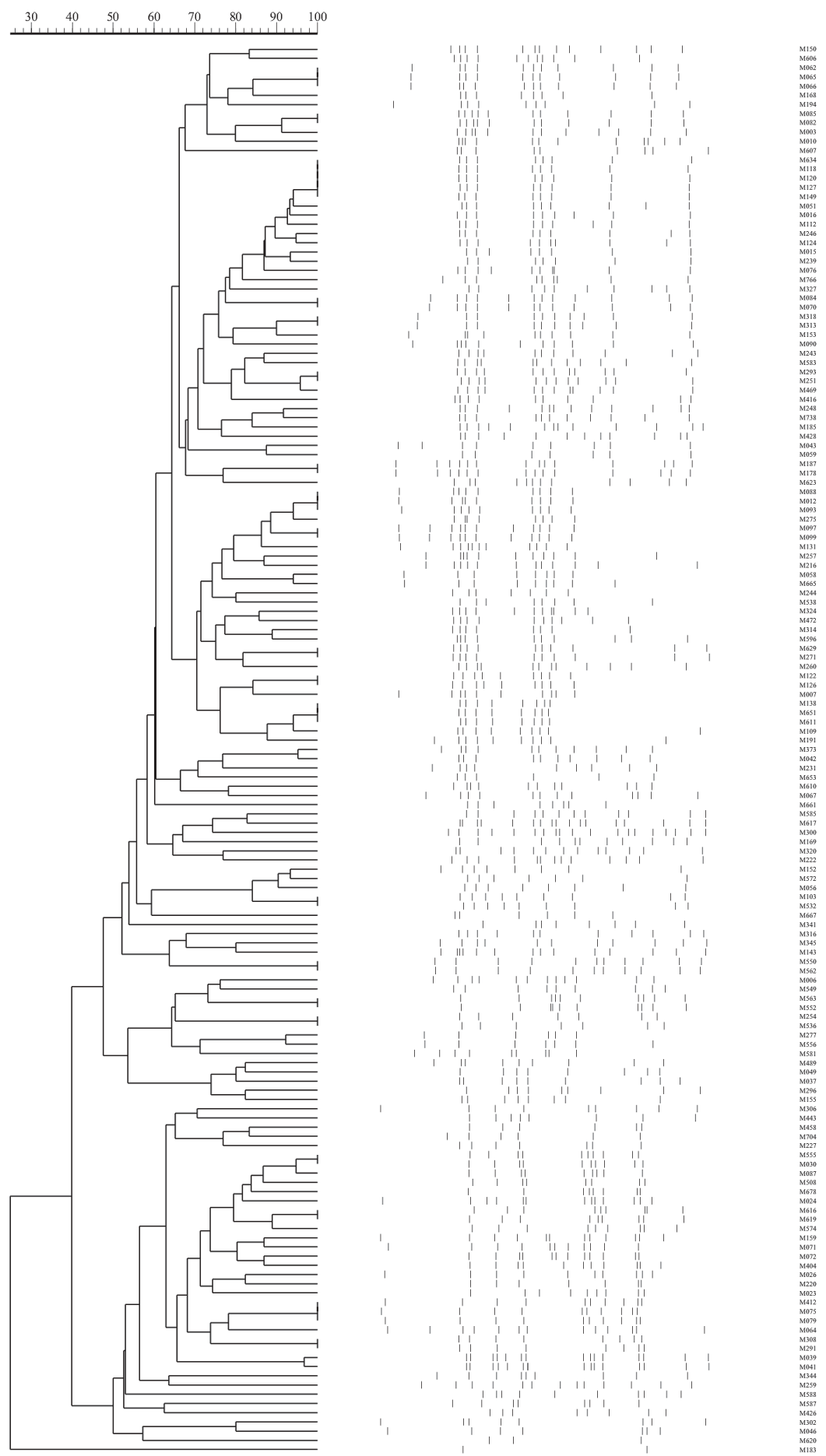

Fig. 2: dendrogram of IS6110-restriction fragment length polymorphism patterns obtained from 153 different patients.

of studies were performed on TB cases from developed countries: These observations, in addition to recent data from patients residing in developing countries, including Brazil, demonstrate the need to evaluate locally, both, epidemiologic and Mtb genotyping characteristics (Palittapongarnpim et al. 1997, Pineda-Garcia et al. 1997, Wilkinson et al. 1997, Dale et al. 1999, Haas et al. 1999, Fandinho et al. 2000, Ferrazoli et al. 2000, Godfrey-Faussett et al. 2000, Suffys et al. 2000, Bruchfeld et al. 2002,
Narayanan et al. 2002, Ferdinand et al. 2003, Easterbrook et al. 2004, Verver et al. 2004, Das et al. 2005).

To our knowledge, this study is the first population based analysis supported by fingerprint data of TB transmission in patients from Rio de Janeiro. Importantly, the study presents some limitations, including the incomplete analysis of the isolates of the TB cases reported during the sampling. This could have influenced our study outcome because clustering level has been directly as- 
TABLE I

Patients and strain characteristics and IS6110-restriction fragment length polymorphism clustering (100\% identity) of Mycobacterium tuberculosis strains from 153 tuberculosis patients (TB)

\begin{tabular}{l}
\hline Possible risk factor \\
\hline Age (in years) \\
$<20$ \\
$20-30$ \\
$31-40$ \\
$41-50$ \\
$>50$
\end{tabular}

\section{Number of cases}

$\begin{array}{llll}\text { Cluster } & \text { OR } & 95 \% \mathrm{CI} & P \text {-value }\end{array}$

Living conditions

Homeless/Shelter/Precarious
(Slums)

House/Apartment

12

Sexual preference

Homosexual/Bisexual

Heterosexual

$\begin{array}{rr}2 & 3 \\ 71 & 76\end{array}$

1.24

$0.14-9.61$

1.00

Education

Illiterate

Elementary incomplete

Elementary complete

High school/College

$\begin{array}{rr}3 & 9 \\ 33 & 65 \\ 6 & 11 \\ 5 & 4\end{array}$

Gender

Male

Female

Permanence in hospital

Yes

No

17

Permanence in police station or prison

Yes

No

Clinical form of TB

Pulmonary

Extrapulmonary

Previous TB treatment

No

Yes

Intravenous drug abuse

Yes

No

Contact with TB

Yes

No

Smear positive cases

Yes

No

3

Drug resistance

(at least one drug)

Yes

No

HIV serology

Positive

Negative

4
44

8

44

87

0.99

0.23-3.90

1.00

BCG scar

Yes

31

58

No

.




\begin{tabular}{lrrrrr}
\hline & \multicolumn{2}{c}{ Number of cases } & & & \\
Possible risk factor & Cluster & Non cluster & OR & $95 \%$ CI & $P$-value \\
\hline Geographic area $^{a}$ & 12 & & & & \\
AP1 & 11 & 29 & & & \\
AP2 & 18 & 48 & & & \\
AP3 & 8 & 16 & 2.74 & $1.02-7.41$ & 0.03 \\
AP5 & & & & \\
\hline
\end{tabular}

$a$ : $\mathrm{AP} 1$ versus $\mathrm{AP} 2+\mathrm{AP} 2+\mathrm{AP} 3+\mathrm{AP} 5$.

TABLE II

Distribution of culture positivity and genotyping of Mycobacterium tuberculosis isolates from patients attended at the "programatic areas" included in the study

\begin{tabular}{lccr}
\hline Area & $\begin{array}{c}\text { Number of culture } \\
\text { positive patients }\end{array}$ & $\begin{array}{c}\text { Number of RFLPs } \\
\text { performed: } \mathrm{n}(\%)\end{array}$ & $\begin{array}{c}\text { Cluster frequency } \\
\mathrm{n}(\%)\end{array}$ \\
\hline AP1 & 62 & $23(37.0)$ & $12(47.8)$ \\
AP2 & 103 & $40(38.8)$ & $11(27.5)$ \\
AP4 & 123 & $66(53.6)$ & $18(27.2)$ \\
AP5 & 61 & $24(39.3)$ & $8(33.3)$ \\
\hline Total & 349 & $153(47.8)$ & $49(32.0)$ \\
\hline
\end{tabular}

$a$ : as defined by $100 \%$ identical RFLP patterns.

sociated with sampling (Glynn et al. 1999, Murray et al. 2002). Fortunately, the clustering levels, observed in the different APs, seem not to be directly related to the sampling fraction size, maintaining our hypothesis that higher clustering in the AP1 region is a measure of more recent transmission. A second limitation could be the time frame of six months for sample collection. In order to avoid missing clustered cases, it has been proposed to collect samples during at least three years study period and to analyze $70 \%$ of all TB cases covered (Vynnycky et al. 2003). Such an approach is however very difficult to establish in an environment such as the city of Rio and few studies, even those performed in developed nations, fulfill this criteria (Barnes \& Cave 2003). However, as the annual risk of infection in the Rio de Janeiro region had not greatly changed during the last decade (Cavalcante et al. 2003), this study may provide reliable positive predictive values for clusters.

Two main characteristics of the Mtb genotypes observed in the present study, a considerable variability and the presence of few low-copy number strains have been described in earlier Brazilian studies (Fandinho et al. 2000, Ferrazoli et al. 2000, Suffys et al. 2000, Telles et al. 2005, Cafrune et al. 2006). This is in concordance with genotype structure observed in most countries but in contrast with studies performed in the Middle East and Asia (Dale et al. 1999, Narayanan et al. 2002, Das et al. 2005). Also, genotype cluster frequency presently observed is similar to the levels of 31 to $48 \%$ described by some investigators from different parts of the world (Wilkinson et al. 1997, Dias et al. 1998, Ferdinand et al. 2003, Das et al. 2005) lower than the 51 to $72 \%$ levels reported in India, Turkey, and some African countries (Godfrey-Faussett et al. 2000, Narayanan et al. 2002, Verver et al. 2004) and higher to the $24 \%$ mentioned in Hong Kong (Chan-Yeung et al. 2003). Notably, the largest cluster was formed by a particular 8-band genotype that takes part of a large clade with very similar RFLP patterns, observed in an earlier study on Brazilian strains from different regions of the country (Suffys et al. 2000).

The most important finding of this study is that IS6110-patterns obtained from strains of patients residing in programmatic area AP1, located in downtown Rio de Janeiro, were significantly associated with clustering. Possibly, poverty, overcrowding, increased human circulation, and higher HIV infection rate among TB registered cases in this area are contributing to recent transmission of TB. This area of the city has traditionally been reported as having higher TB incidence than other parts (Cavalante et al. 2003). Higher clustering, in some specific urban areas with higher TB incidence, has described in other big cities (Verver et al. 2004). Besides residence, no other case or parasite characteristics were associated with genotype clustering. Not all epidemiologic studies combining conventional and molecular approaches identify risk factors for TB transmission but among those frequently reported are sputum smear positivity (Verver et al. 2004), history of imprisonment or hospitalization (Narayanan et al. 2002), relapse (Narayanan et al. 2002), failure of anti-TB treatment (GodfreyFaussett et al. 2000), young age, and belonging to social networks. As related before, besides the absence of risk factors being due to sampling, differences in regional (geographic, whether), population (ethnic, behavioral), and bacterial population-associated characteristics also influence transmission patterns; indeed, some assumptions on the disease may be true only in a particular environment (Cohn et al. 1998). Indeed, HIV-infection, drug-resistant forms of TB and homelessness, factors often associated with more intense TB transmission in developed nations, does not seem to influence clustering as much in poor nations. In the latter, and supporting our data, clustering tended to occur more frequently among isolates from patients residing in areas where TB incidence is higher (Small et al. 1994, Barnes \& Cave 2003, Lambregts-van Weezen-beek et al. 2003, Munsiff et al. 2003, Seidler et al. 2004). 
Although differences in Mtb genotype clustering is the basis for definition of risk factors for TB, confirmation of transmission between patients belonging to clusters enforces the value of this statement. In the present evaluation, this could not be performed due to the design of the questionnaire and lack of funds for re-interviewing selected cases but in studies where this was performed, an epidemiologic link ranging from 16 to $30 \%$ was observed among the clustered cases, demonstrating the gain in information when using Mtb genotyping but suggesting also the need to better investigate the accuracy of the procedure in general (Wilkinson et al. 1997, Montoro et al. 1998, Chan-Yeung et al. 2003, Das et al. 2005).

Cluster level, observed during molecular epidemiologic studies, is dependent on the stringency of analysis: small changes of IS6110 banding patterns occur during transmission and can lead to underestimation of clustering levels and missing transmission chains or cases (Warren et al. 2002). Lowering cluster definition stringency did not influence the outcome of the present study. Nonetheless, many reports including molecular data for TB studies use a secondary typing to increase the accuracy of indicating epidemiological links. In the case of IS6110-RFLP patterns, this is considered necessary mainly, when dealing with Mtb populations presenting a high proportion of low-copy number strains (Rhee et al. 2000). Besides, data exist on increased accuracy of typing data when using a secondary technique upon interpretation of IS6110-RFLP clusters even of high copy number patterns (Yang et al. 2001). When considering only strains with identical IS6110-RFLP and DRE-PCR patterns as part of a cluster (85\% of analyzed IS6110RFLP clustered strains), the significantly higher cluster level in AP1 was maintained. Differentiation by DREPCR of strains with clustered IS6110-RFLP patterns was generally due to single band differences and could be related to the presence of a sub-population detected by PCR but not by Southern-based techniques. The difference between DRE-PCR patterns of some of the strains forming the biggest RFLP cluster was more pronounced but we suspect that this RFLP pattern, besides being also the most frequent in some other studies on Brazilian Mtb genotypes, represents a dominant and deeply rooted clade and on its own does not always indicate recent transmission (Suffys et al. 2000). This is now under investigation.

Concluding, limitations in sampling is probably a main factor for lack of definition of risk factors for TB transmission in the present study but the Mtb fingerprinting data are in agreement with the indication of the central part of Rio de Janeiro being a more problematic area of TB. This encourages the use of fingerprinting in this setting in a well designed prospective epidemiologic study, as being realized for evaluation of the DOTS program from the City TB Program, implemented in this area in 1998 (Cavalcante et al. 2003).

\section{ACKNOWLEDGEMENTS}

To Oscar Berro of the Central Laboratory Noel Nutels for providing the necessary laboratory evaluation, Betina Durovni of the Municipal Secretary of Health of Rio de Janeiro for providing the necessary access to the Health Unit and identifying the key personnel to take part in this study. To Cristina Diniz Dantas and Mariana da Silva Maia for patient care and data collection.

\section{REFERENCES}

Barnes PF, Cave MD 2003. Molecular epidemiology of tuberculosis. New Engl J Med 349: 1149-1156.

Bennet S, Woods T, Lyianage WM, Smith DL 1991. A simplified general method for cluster-sample surveys of health in developing countries. World Health Stat Q 44: 98-106.

Brasil 1994. Manual de Bacteriologia da Tuberculose, 2 ${ }^{\underline{a}}$ ed., Ministério da Saúde, Funasa, Rio de Janeiro.

Bruchfeld J, Aderaye G, Palme IB, Bruchfeld J, Aderaye G, Palme IB, Bjorvatn B, Britton S, Feleke Y, Kallenius G, Lindquist L 2002. Molecular epidemiology and drug resistance of Mycobacterium tuberculosis isolates from Ethiopian pulmonary tuberculosis patients with and without human immunodeficiency virus infection. J Clin Microbiol 40: 1636-1643

Cafrune PI, Riley LW, Possuelo LG Valim ARM, Borges M, Ribeiro MO, Rossetti MLR, Zaha A 2006. Recent transmission of tuberculosis involving retired patients. J Infect 53: 370-376.

Caminero JA, Pena MJ, Campos-Herrero MI, Rodriguez JC, Afonso O, Martin C, Pavon JM, Torres MJ, Burgos M, Cabrera P, Small PM, Enarson DA 2001. Exogenous reinfection with tuberculosis on a European island with a moderate incidence of disease. Am J Respir Crit Care Med 163: 717-720.

Cavalcante SC, Soares E, da Rocha M, de Oliveira J, Dias S, Pacheco A 2003. A implantação da estratégia DOTS na cidade do Rio de Janeiro. Pulmão RJ: 12.

Chan-Yeung M, Tam CM, Wong H, Leung CC, Wang J, Yew WW, Lam CW, Kam KM 2003. Molecular and conventional epidemiology of tuberculosis in Hong Kong: a populationbased prospective study. J Clin Microbiol 41: 2706-2708.

Cohn DL, O'Brien RJ 1998. The use of restriction fragment length polymorphism (RFLP) analysis for epidemiological studies of tuberculosis in developing countries. Int J Tuberc Lung Dis 2: 1626.

Cowan LS, Diem L, Monson T, Wand P, Temporado D, Oemig TV, Crawford JT 2005. Evaluation of a two-step approach for large-scale, prospective genotyping of Mycobacterium tuberculosis isolates in the United States. J Clin Microbiol 43: 688-695.

Dale JW, Mat Nor R, Ramayah S, Tang TH, Zainuddin ZF 1999. Molecular epidemiology of tuberculosis in Malaysia. J Clin Microbiol 37: 1265-1268.

Das SD, Narayanan S, Hari L, Hoti SL, Thangathurai RK, Charles N, Jaggarajamma K, Narayanan PR 2005. Differentiation of highly prevalent IS6110 single-copy strains of M. tuberculosis from a rural community in South India with an ongoing DOTS programme. Infec Genet Evol 5: 65-77.

Easterbrook PJ, Gibson A, Murad A, Lamprecht D, Ives N, Ferguson A, Lowe O, Mason P, Ndudzo A, Taziwa A, Makombe R, Mbengeranwa L, Sola C, Rastogi N, Drobniewski F 2004. High rates of clustering of strains causing tuberculosis in Harare, Zimbabwe: a molecular epidemiological study. J Clin Microbiol 42: 4536-4544

Fandinho FCO, Kritski AL, Hofer C, Junior Conde H, Ferreira RM, Saad MH, Silva MG, Riley LW, Fonseca LS 2000. RFLP patterns and risk factors for recent tuberculosis transmission among hospitalized tuberculosis patients in Rio de Janeiro, Brazil. Trans R Soc Trop Med Hyg 94: 271-275.

Ferdinand S, Sola C, Verdol B, Legrand E, Goh KS, Berchel M, Aubert A, Timote M, Joseph P, Pape JW, Rastogi N 2003. Molecular characterization and drug resistance patterns of 
strains of Mycobacterium tuberculosis isolated from patients in an AIDS counseling center in Port-au-Prince, Haiti: a 1-year study. J Clin Microbiol 41: 694-702.

Ferrazoli L, Palaci M, Marques LRM, Jamal LF, Afiune JB, Chimara E, Martins MC, Silva Telles MA, Oliveira CA, Palhares MC, Spada DT, Riley LW 2000. Transmission of tuberculosis in an urban setting in Brazil. Int J Tuber Lung Dis 4: 18-25.

Glynn JR, Vynnycky E, Fine PE 1999. Influence of sampling on estimates of clustering and recent transmission of $M$. tuberculosis derived from DNA fingerprinting techniques. Am J Epidemiol 149: 366-371.

Godfrey-Faussett P, Sonnenberg P, Shearer SC, Bruce MC, Mee C, Morris L, Murray J 2000. Tuberculosis control and molecular epidemiology in a South African gold-mining community. Lancet 356: 1066-1071.

Haas WH, Engelmann G, Amthor B, Shyamba S, Mugala F, Felten M, Rabbow M, Leichsenring M, Oosthuizen OJ, Bremer HJ 1999. Transmission dynamics of tuberculosis in a high-incidence country: prospective analysis by PCR DNA fingerprinting. J Clin Microbiol 37: 3975-3979.

Kent PT, Kubica GP 1985. Public Health Mycobacteriology. A Guide for a Level III Laboratory, Centers for Disease Control, Atlanta.

Lambregts-van Weezenbeek CSB, Sebek MG, van Gerven PJ, de Vries G, Verver S, Kalisvaart NA, van Soolingen D 2003. Tuberculosis contact investigation and DNA fingerprint surveillance in The Netherlands: 6 years' experience with nation-wide cluster feedback and cluster monitoring. Int $J$ Tuberc Lung Dis 7: S463-S470.

Lan NTN, Lien HTK, Tung LB, Borgdorff MW, Kremer K, van Soolingen D 2003. Mycobacterium tuberculosis Beijing genotype and risk for treatment failure and relapse Vietnam. Emerg Infect Dis 9: 1633-1635.

Ministry of Health BNSAp 2004. AIDS drug policy. http:// www.aids.gov.br/assistencia/aids_drugs_policy.htm. Access April 2004.

Montoro E, Valdivia J, Leão SC 1998. Molecular fingerprinting of Mycobacterium tuberculosis isolates obtained in Havana, Cuba, by IS6110 restriction fragment length polymorphism analysis and by the double-repetitive-element PCR method. J Clin Microbiol 36: 3099-3102.

Munsiff SS, Nivin B, Sacajiu G, Mathema B, Bifani P, Kreiswirth $\mathrm{BN}$ 2003. Persistence of a highly resistant strain of tuberculosis in New York City during 1990-1999. J Inf Dis 188: 356-363.

Murray M, Alland D 2002. Methodological problems in the molecular epidemiology. Am J Epidemiol 155: 565-571.

Narayanan S, Das S, Garg P, Hari L, Rao VB, Frieden TR, Narayanan PR 2002. Molecular epidemiology of tuberculosis in a rural area of high prevalence in South India: implications for disease control and prevention. J Clin Microbiol 40: 47854788.

Palittapongarnpim P, Luangsook P, Tansuphaswadikul S, Chuchottaworn C, Prachaktam R, Sathapatayavongs B 1997. Restriction fragment length polymorphism study of Mycobacterium tuberculosis in Thailand using IS6110 as probe. Int J Tuberc Lung Dis 1: 370376.

Pineda-Garcia L, Ferrera A, Hoffner SE 1997. DNA Fingerprinting of Mycobacterium tuberculosis strains from patients with pulmonary tuberculosis in Honduras. J Clin Microbiol 35: 2393-2397.

Rhee JT, Tanaka MM, Behr MA, Agasino CB, Paz EA, Hopewell PC, Small PM 2000. Use of multiple markers in populationbased molecular epidemiologic studies of tuberculosis. Int $J$ Tuberc Lung Dis 4: 111-1119.

Seidler A, Nienhaus A, Diel R 2004. The transmission of tuberculosis in the light of new molecular biological approaches. Occup Environ Med 61: 96-102.

Small PM, Hopewell PC, Singh SP 1994. The epidemiology of tuberculosis in San Francisco: a population-based study using conventional and molecular methods. N Engl J Med 330: 1703-1709.

Suffys PN, Ivens de Araujo ME, Rossetti ML, Zahab A, Barroso EW, Barreto AM, Campos E, van Soolingen D, Kremer K, Heersma H, Degrave WM 2000. Uselfulness of IS6110-restriction fragment length polymorphism typing of Brazilian strains of Mycobacterium tuberculosis and comparison with an international fingerprint database. Res Microbiol 151: 343-351.

Telles MA, Ferrazoli L, Waldman EA, Giampaglia CM, Martins MC, Ueki SY, Chimara E, Silva CA, Cruz V, Waldmann CC, Heyn I, Hirono IU, Riley LW 2005. A population-based study of drug resistance and transmission of tuberculosis in an urban community. Int J Tubercle Lung Dis 9: 970-976.

van Embden JDA, Cave MD, Crawford JT, Dale JW, Eisenach KD, Gicquel B, Hermans P, Martin C, McAdam R, Shinnick TM 1993. Strain identification of Mycobacterium tuberculosis by DNA fingerprinting: recommendations for a standardized methodology. J Clin Microbiol 31: 406-409.

van Rie A, Warren RM, Beyers N, Gie RP, Classen CN, Richardson M, Sampson SL, Victor TC, van Helden PD 1999. Transmission of a multi drug resistant $M$. tuberculosis strain resembling strain W among noninstitutionalized, human immunodeficiency virus seronegative patients. J Infect Dis 180: 1608-1615.

Verver S, Warren RM, Munch Z, Vynnycky E, van Helden PD, Richardson M, van der Spuy GD, Enarson DA, Borgdorff MW, Behr MA, Beyers N 2004. Transmission of tuberculosis in a high incidence urban community in South Africa. Int J Epidemiol 33: 351-357.

Vynnycky E, Borgdorff MW, van Soolingen D, Fine P 2003. Annual $M$. tuberculosis risk and interpretation of clustering statistics. Emerg Infect Dis 9: 176-183.

Warren RM, van der Spuy GD, Richardson M, Beyers N, Booysen C, Behr MA, van Helden PD 2002. Evolution of the IS6110based restriction fragment length polymorphism pattern during the transmission of Mycobacterium tuberculosis. J Clin Microbiol 40: 1277-1282.

WHO report 2006. Global tuberculosis control: surveillance, planning, financing. World Health Organization, Geneva, (WHO/ HTM/TB/2005.349).

Wilkinson D, Pillay M, Crump J, Lombard C, Davies GR, Sturm AW 1997. Molecular epidemiology and transmission dynamics of Mycobacterium tuberculosis in rural Africa. Trop Med Int Health 2: 747-53

Yang ZH, Bates JH, Eisenach KD, Cave MD 2001. Secondary typing of Mycobacterium tuberculosis isolates with matching IS6110 fingerprints from different geographic regions of United States. J Clin Microbiol 39: 1691-1695. 